\title{
Resultados preliminares del método RULER en la inteligencia emocional y el compromiso laboral de profesores Españoles
}

\section{Ruth Castillo-Gualda ${ }^{1}$, Valme García ${ }^{2}$, Mario Pena ${ }^{3}$, Arturo Galán ${ }^{4}$ y Marc A. Brackett ${ }^{5}$}

${ }^{1}$ Facultad de Educación y Facultad de Salud, Universidad Camilo José Cela, Madrid

${ }^{2}$ Colegio Internacional Aravaca, Madrid, España

${ }^{3}$ Dpto. MIDE II (OEDIP) Universidad Nacional de Educación a Distancia, Madrid, España

${ }^{4}$ Dpto. MIDE I Universidad Nacional de Educación a Distancia, Madrid, España

${ }^{5}$ Yale Center for Emotional Intelligence, New Haven, EEUU.

\section{España / EEUU}

Correspondencia: Ruth Castillo Gualda. Urb. Villafranca del Castillo, Calle Castillo de Alarcón, 49, 28692.

Villanueva de la Cañada, Madrid. E-mail: rcastillo@ucjc.edu

(C) Universidad de Almería and Ilustre Colegio Oficial de la Psicología de Andalucía Oriental (Spain) 


\section{Resumen}

Introducción. El objetivo de este estudio fue evaluar el impacto de un programa de educación socio-emocional, el método RULER, en Inteligencia emocional como habilidad, evaluada por el test de inteligencia emocional Mayer-Salovey-Caruso (MSCEIT), así como en el compromiso y estrés laboral.

Método. Participaron en el mismo dos escuelas privadas de la Comunidad Autónoma de Madrid (España). Una vez que las escuelas fueron asignadas a la condición experimental o control aleatoriamente, un total de cincuenta y cuatro profesores participaron en la presente investigación, grupo RULER ( $n=32)$, grupo control $(n=22)$.

Resultados. El análisis multivariado de covarianza (MANCOVA) reveló que tras 24 horas de formación repartidas a lo largo de tres meses, el grupo RULER obtuvo resultados significativamente mejores en las habilidades de percepción, comprensión y regulación emocional, así como en variables relacionadas con la satisfacción y compromiso en su trabajo docente, en comparación con el grupo control.

Discusión y conclusiones. Estos hallazgos amplían la literatura sobre el impacto de los programas científicos en el desarrollo de los recursos personales, así como la competencia social y emocional en población adulta. El artículo también discute las implicaciones prácticas y líneas futuras de la intervención socio-emocional en población adulta, para el desarrollo de habilidades personales y profesionales, como factores clave para una óptima labor docente.

Palabras Clave: educación socio-emocional, inteligencia emocional, profesores, intervención socio-emocional, desarrollo profesional. 


\begin{abstract}
Introduction. The goal of this study was to assess the effectiveness of a socio-emotional learning program, RULER, on enhancing both the emotional intelligence and work-related outcomes in Spanish teachers. Measures included: Ability emotional intelligence, assessed by the Mayer-Salovey-Caruso Emotional Intelligence Test (MSCEIT) and work-related outcomes, such as engagement and burnout.
\end{abstract}

Method. Two private schools from the Autonomous Community of Madrid participated in the study. Once the schools were assigned to experimental or control condition randomly, a total of fifty-four teachers served in the present research, $n=32$ teachers participated in the SEL program, $n=22$ teachers remained as control group.

Results. Multivariate analysis of covariance (MANCOVA) revealed that the SEL group obtained significantly better results in the areas of emotional perception, understanding, and regulation, as also in those related to satisfaction, and commitment to their work, after twentyfour hours of SEL training, across three months, compared with control group.

Discussion and Conclusion: These findings expand the literature on the impact of scientificbased programs in the development of personal resources and social and emotional competence in adult population. The paper also discusses practical implications and future research of socio-emotional interventions among adult population, for the development of personal and professional skills, as key factors for an optimal teaching practice.

Keywords: socio-emotional learning, emotional intelligence, teachers, socio-emotional intervention, professional development. 


\section{Introducción}

La profesión docente es esencialmente emocional. La manera en que los educadores procesen y gestionen sus propias emociones, así como la forma en la que se relacionen con las emociones de sus alumnos, es el elemento diferenciador de un entorno de enseñanza y aprendizaje óptimo (Yin, Lee, Zhang y Jin, 2013; Kurki, Järvenoja, Järvelä, y Mykkänen, 2016). Esta característica la convierte, a su vez, en una de las profesiones con mayores niveles de desgaste profesional y agotamiento (Betoret, 2009). La elevada responsabilidad inherente a la profesión docente, así como la respuesta efectiva a las necesidades académicas y personales del alumnado, puede desembocar en insatisfacción laboral, con el consiguiente riesgo de estrés laboral o burnout (Chang, 2013) y sus importantes implicaciones en la salud mental de los profesionales (Aloe, Amo y Shanahan, 2014). Sin embargo, las competencias emocionales para hacer frente a estas demandas, pueden ser un factor decisivo en su desempeño como docentes. Es más, las demandas contextuales pueden incluso relacionarse con el bienestar emocional y laboral, si el profesional percibe que cuenta con los recursos personales necesarios para afrontarlas (Bakker, Hakanen, Demerouti, y Xanthopoulou, 2007; De Jonge y Dormann, 2006). Estas competencias vienen a ser conceptualizadas bajo el término Inteligencia Emocional (IE), definido como el conjunto de habilidades mentales que facilitan el procesamiento de la información emocional (Mayer y Salovey, 1997). El adecuado reconocimiento, uso y conocimiento de las emociones tanto en uno mismo, como en los demás, así como su manejo efectivo, se relaciona con las capacidades cognitivas para hacer frente a situaciones de intensidad emocional. De este modo se favorece la resolución exitosa de conflictos, las relaciones sociales más saludables y el manejo efectivo del estrés (Mayer, Roberts, y Barsade, 2008). Así, las citadas habilidades y, en concreto, la regulación emocional, son una fuente importante de recursos personales internos, que se asocian a un mayor ajuste psicológico y bienestar emocional (Extremera y Rey, 2016).

Numerosas investigaciones han comprobado cómo las habilidades de IE se relacionan con un mayor desempeño, éxito profesional, mejores condiciones laborales y mejores estrategias de afrontamiento, en profesionales de diferentes colectivos (Blanco-Donoso, Demerouti, Garrosa, Moreno-Jimenez y Carmona, 2015). Proporcionan mayor tolerancia al estrés y, por tanto, mayor compromiso o emocionalidad positiva en el trabajo (Blanco-Donoso et al., 2015). En contraste, aquellos profesionales con menores habilidades emocionales se caracterizan por realizar una interpretación más negativa de las demandas del entorno, de su propia 
actividad y de su percepción de auto-eficacia, por lo que ponen en marcha estilos de afrontamiento más desadaptativos (Johnston, Hansen, Birney y Stough, 2010).

Estas asociaciones han sido, adicionalmente, constatadas en muestras de profesores. Las habilidades de IE facilitan relaciones más afectivas, expectativas más positivas y elicitan respuestas más óptimas por parte de su entorno (Brackett, Palomera, Mojsa-Kaja, Reyes, y Salovey, 2010). En general, estas habilidades pueden favorecer el manejo efectivo de situaciones desafiantes, optimizar las relaciones, reconocer las limitaciones propias, modelar e influir el comportamiento/rendimiento de los alumnos, atender a las necesidades personales de los mismos, así como facilitar el planteamiento y alcance de objetivos académicos adecuados (Saklofske, Austin, Mastoras, Beaton y Osborne, 2012). En consecuencia, estos procesos -tanto emocionales como sociales- parecen estar en la base de un mayor bienestar laboral y desempeño óptimo por parte de estos profesionales (Rey, Extremera, y Pena, 2016).

\section{Los Programas para el desarrollo de la Inteligencia Emocional}

Los programas para el desarrollo de las habilidades de IE, llamados en la literatura científica, programas de educación emocional y social (SEL), se basan en la promoción y mejora de cinco conjuntos de competencias emocionales, cognitivas y comportamentales: (a) habilidades de autoconsciencia; (b) manejo emocional; (c) consciencia social; (d) relaciones interpersonales y (e) toma de decisiones responsable (Durlak, Domitrovich, Weissberg, y Gullotta, 2015). En este sentido, el modelo de habilidad de la IE (Mayer y Salovey, 1997) conceptualiza e integra dichas competencias en un mismo constructo científicamente contrastado y fácilmente evaluable (Mayer, Roberts y Barsade, 2008). Los programas SEL se basan en la promoción de aspectos emocionales y sociales desde edades tempranas, con el objetivo de prevenir problemas y desarrollar mecanismos para un ajuste psicológico óptimo (Durlak, Weissberg, Dymnicki, Taylor y Schellinger, 2011).

\section{Efectividad de los programas SEL en el alumnado}

La eficacia de los programas SEL ha sido puesta de manifiesto en diversos estudios y se ha visto respaldada con las recientes revisiones realizadas sobre estas iniciativas (Durlak et al., 2011; Durlak et al., 2015). En concreto, existe una amplia evidencia científica que muestra el impacto positivo de estas iniciativas en el desarrollo de las competencias socio-emocionales en la resolución de conflictos y clima de clase (Hagelskamp, Brackett, Rivers, y Salovey, 
2013; Reyes, Brackett, Rivers, Elbertson y Salovey, 2012). Lo mismo sucede en el ajuste psicológico y la salud mental de los estudiantes (Ruiz-Aranda, Castillo, Salguero, Cabello, Fernández-Berrocal y Balluerka, 2012), en el desarrollo de las conductas prosociales y reducción de comportamientos agresivos (Castillo, Cabello, Herrero-Lázaro, Rodríguez-Carvajal y Fernández-Berrocal, en revisión; Castillo, Salguero, Fernández-Berrocal y Balluerka, 2013) y en el incremento del rendimiento académico y los resultados escolares, en comparación con los alumnos que no recibieron este tipo de programas (Brackett, Rivers, Reyes y Salovey, 2012).

\section{Los programas SEL dirigidos a la comunidad educativa en general}

Sin embargo, el impacto de este tipo de iniciativas SEL no tiene por qué limitarse únicamente al alumnado, sino que puede extenderse también a los profesores y a la comunidad educativa en general. Es más, se asume que los profesionales de la educación deben contar con un abanico de competencias socio-emocionales necesarias para ser un modelo efectivo de manejo emocional frente a sus estudiantes, resolver situaciones novedosas o dar respuesta a escenarios conflictivos (Jennings y Greenberg, 2009). Erróneamente, parece que estas competencias son características personales que los profesores tienen la suerte o no de poseer, o como mucho tienen la oportunidad de desarrollar a lo largo de su carrera profesional, pero que poco puede hacerse por desarrollarlas mediante entrenamiento formal (Pérez-Escoda et al., 2012; Talvio et al., 2013). Sin embargo, un área que parece prometedora y que hasta ahora ha sido escasamente evidenciada, es el beneficio que pueden obtener los profesionales de la educación al recibir programas de intervención para el desarrollo y la capacitación de habilidades de IE (Jennings y Greenberg, 2009), no sólo por el impacto directo en el desarrollo óptimo de su alumnado y en la mejora de su rendimiento, sino también por el efecto de las citadas habilidades en el desarrollo personal y profesional de los propios profesores. En concreto, en base a investigaciones previas, dichas competencias pueden facilitar decisivamente su crecimiento personal, su bienestar emocional, y el desarrollo de la satisfacción y compromiso en su labor docente, aspectos muy relevantes para la promoción de entornos educativos más efectivos (Brackett et al., 2010).

Entre los efectos más significativos de este tipo de intervenciones, se encuentra el impacto sobre la calidad de las relaciones interpersonales o el desarrollo de un clima positivo en clase que implica tanto a estudiantes, como a docentes. Así, se ha demostrado que aquellas aulas que integran programas SEL se caracterizan por alcanzar climas educativos más positivos para el aprendizaje, mayores niveles de motivación tanto en profesores como en alumnos 
y mejores relaciones entre unos y otros (Castillo, Fernández-Berrocal, y Brackett, 2013; Roeser, Skinner, Beers, y Jennings, 2012; Talvio et al., 2013). Investigaciones previas además muestran como este tipo de programas logran aumentar y desarrollar las habilidades de IE y auto-eficacia en profesorado (Pool y Qualter, 2012). Estos resultados suponen por tanto, una importante vía con la que potenciar la efectividad y calidad del proceso enseñanzaaprendizaje (Natahnson, Rivers, Flynn y Brackett, 2016). De hecho, uno de los programas SEL dirigido a la comunidad educativa en general, cuya principal premisa es el entrenamiento y la mejora de numerosas variables relevantes para el bienestar personal y profesional, es el método RULER (Bracket y Rivers, 2013), que se centra en un enfoque para la promoción de las habilidades de IE y está científicamente basado en el modelo teórico de la IE como habilidad (Mayer y Salovey, 1997). Dicho método está compuesto por una serie de herramientas y prácticas educativas que se integran en el curriculum del centro (Natahnson, et al., 2016). No obstante, todavía son muy escasos los resultados referentes a la efectividad de los programas SEL en población adulta (Pool y Qualter, 2012), y todavía más escaso en contextos educativos españoles, por lo que se hace necesario profundizar en las posibles implicaciones y beneficios durante la etapa adulta

\section{Evaluación de las competencias socio-emocionales con el MSCEIT}

Uno de los problemas de la investigación en el área de intervención SEL, es el relativo a las medidas de evaluación y los enfoques teóricos desde los que se sustentan las descritas competencias socio-emocionales. Concretamente, el modelo de habilidad de la IE concibe estas competencias como destrezas mentales. Por ello, deben ser medidas por tareas de ejecución, pues esta concepción requiere de técnicas que evalúen la ejecución real de la persona ante una determinada situación. Los resultados encontrados con el MSCEIT confirman la naturaleza del constructo de IE como una capacidad mental claramente definida, con una adecuada validez estructural, tanto de los factores o ramas que componen la medida, como de la puntuación global (Mayer, Salovey y Caruso, 2002; Extremera y Fernández-Berrocal, 2009). Adicionalmente, el análisis factorial confirmatorio ha mostrado una adecuación al modelo jerárquico original de la IE planteado por los autores en una amplia muestra española (Sánchez-García, Extremera y Fernández-Berrocal, 2016). Por todo ello, la conceptualización de estas habilidades de IE incluidas bajo el modelo teórico descrito, permite trascender de los conceptos más estables incluidos teóricamente en los modelos mixtos (e.g., Bar-On, 2006; Petrides y Furnham, 2003), como son la motivación, el optimismo o la tolerancia al estrés, 
con el fin de analizar la contribución específica de las citadas competencias, de manera específica (Mayer, Roberts, y Barsade, 2008). Por ello, el MSCEIT supone una forma de evaluar tales competencias de manera precisa y objetiva, así como de observar el impacto de programas de intervención con medidas de ejecución real.

\section{Objetivos e hipótesis}

El objetivo de esta investigación es comprobar la efectividad del método RULER —un programa basado en el modelo de habilidad de IE (Mayer y Salovey, 1997)- en el contexto de evaluación de programas SEL. Se hipotetiza que el método RULER tendrá un efecto positivo en el desarrollo de las habilidades de IE (evaluadas mediante una tarea de ejecución real), así como en la satisfacción laboral, el nivel de compromiso o engagement docente y la reducción de los niveles de burnout o estrés laboral.

\section{Método}

\section{Participantes}

La muestra estuvo formada por 54 profesionales (profesores y directivos, de los que el $79 \%$ son mujeres), pertenecientes a dos centros educativos de la Comunidad de Madrid: centro 1 (Experimental) y centro 2 (Control). Ambos centros educativos son de titularidad privada y están situados en la misma población, una zona residencial de clase socioeconómica media-alta de Madrid (España). Son dos centros escolares con proyectos curriculares afines, comparten el mismo consultor educativo y tienen un modelo metodológico similar: utilizan estrategias metodológicas basadas en las Inteligencia Múltiples de Gardner (Gardner, 1983) y en el trabajo cooperativo de Kagan (Kagan, 1994). Los profesores de edades comprendidas entre 23 y 59 años $(M=35,98$; DT=8,45), de los cuales el $27 \%$ pertenecían a la etapa de educación infantil (3-5 años), el 51\% a la de primaria, el 12\% a la de secundaria, el 6\% impartía docencia en primaria/secundaria y el $4 \%$ restante formaba parte del equipo de orientación y dirección. Una vez elegido el centro educativo, los profesores pertenecientes al centro experimental, recibieron el programa de intervención. El grupo de profesores que recibió la formación RULER fueron un total de 32 participantes (27 mujeres), con edades comprendidas entre los 23 y 56 años $(M=34,06$; DT=7,16). Los años de experiencia iban de 1 a 20 años $(M=7,54$; DT=4,54). Similarmente, el grupo control estuvo compuesto por 22 participantes (16 mujeres), con edades comprendidas entre los 25 y 59 años $(M=39,41$; DT=9,60). Los años de ex- 
periencia iban de 4 a 15 años $(M=9,08$; DT=3,53). Todos los participantes en la investigación, dieron su consentimiento informado para la evaluación de las variables objeto de estudio.

\section{Instrumentos}

Inteligencia Emocional (MSCEIT; Mayer, Salovey y Carruso, 2002), adaptación en castellano (Extremera y Fernández-Berrocal, 2009). Esta medida de ejecución de la IE evalúa cada una de las ramas del modelo de habilidad de la IE mediante 141 ítems, organizados en las 4 ramas o áreas principales del constructo: Percepción Emocional, medida a través de 2 subescalas que evalúan la identificación de emociones a través de expresiones faciales e imágenes. Facilitación Emocional que incluye otras 2 subescalas que evalúan en qué medida las emociones pueden ser empleadas en diferentes situaciones y la forma en que éstas pueden asociarse a sensaciones. Comprensión Emocional, incluye dos subescalas que evalúan el conocimiento acerca de la combinación de emociones, causas y consecuencias, así como la evolución y el desarrollo de las mismas. Regulación Emocional, formada por otras 2 subescalas que evalúan el uso de estrategias de regulación emocional eficaces para manejar las emociones propias y las de los demás. La particularidad de esta escala recae en que los resultados corresponden a la ejecución real de la persona ante diferentes tareas y situaciones emocionales. Las respuestas correctas son corregidas según un criterio de baremación. Los coeficientes de fiabilidad para las cuatro ramas van desde 0,71 a 0,92. (Mayer, Salovey, et al., 2002; Extremera y Fernández-Berrocal, 2009; Sánchez-García, Extremera y Fernández-Berrocal, 2016).

Compromiso en el Trabajo. Se utilizó la versión en castellano de la Utrecht Work Engagement Scale (UWES; Schaufeli, Salanova, González-Romá, y Bakker, 2002). En el instrumento se define el compromiso laboral como un estado mental positivo en el puesto de trabajo. Se trata de una escala Likert de 7 puntos $(0=$ nunca a $6=$ siempre $)$ compuesta por 15 ítems distribuidos en tres factores: Vigor, definido como altos niveles de energía y resiliencia mental mientras se trabaja, así como la disposición para el esfuerzo en el propio trabajo y la persistencia ante las dificultades. Dedicación, hace referencia al sentido de significado, inspiración, orgullo y desafío en el trabajo; y Absorción, dimensión relacionada con la completa concentración y compromiso en el trabajo, donde parece que se detienen las dificultades y el tiempo pasa rápidamente. En nuestra muestra de estudio obtuvimos unos índices de $\alpha=0,87$ (Vigor); $\alpha=0,90$ (Dedicación) y $\alpha=0,87$ (Absorción). 
Burnout. Para evaluar el desgaste o estrés laboral se utilizó la versión en castellano del Maslach Burnout Inventory (MBI-ES; Maslach, Jackson, y Leiter, 1996). Se trata de una escala Likert de 22 ítems de seis grados (1= nunca; 6 = siempre $)$. Está diseñado para evaluar tres aspectos del burnout: Agotamiento Emocional, refleja el grado de estrés laboral. Despersonalización, evalúa el distanciamiento cognitivo, indiferencia o actitud cínica mientras se trabaja y Realización Personal, que se relaciona con los sentimientos de eficacia y logro en el trabajo (a diferencia de las dos dimensiones anteriores, los niveles bajos de esta corresponden a altos niveles de burnout. Este instrumento ha mostrado adecuados índices psicométricos (Seisdedos, 1997). En nuestra muestra de estudio obtuvimos unos índices de $\alpha=0,83$ (Agotamiento Emocional); $\alpha=0,48$ (Despersonalización) y $\alpha=0,90$ (Realización personal).

\section{Procedimiento}

El presente estudio es un estudio cuasi-experimental pre-post con un grupo experimental (centro 1) y un grupo control (centro 2). El grupo de investigación realizó una propuesta formal a la dirección de los centros educativos para requerir su participación en el estudio. Los dos centros decidieron voluntariamente participar. Aleatoriamente, se asignó la condición experimental al centro 1 y el centro 2 sirvió como grupo control. Los datos pertenecientes al pre-test fueron recogidos antes de iniciar la formación RULER, tanto para la muestra experimental, como para la muestra control. Los datos post-test fueron completados un año después, tanto para el grupo experimental, como para el grupo control.

\section{Intervención: El Metodo RULER}

La idea de que las emociones son esenciales para el aprendizaje, la toma de decisiones, el éxito académico, personal y social, representa la base sobre la que se construye el método RULER. El método RULER es una intervención SEL basada en el modelo teórico de las cuatro ramas de IE descrito anteriormente (Mayer y Salovey, 1997). La formación persiguió los siguientes objetivos: (a) desarrollar las habilidades emocionales de los docentes para involucrar a los estudiantes en el aprendizaje; (b) aprovechar las habilidades emocionales para convertirse en buenos profesionales educativos, mejorar las relaciones personales/profesionales (c) hacer frente al estrés; y (d) asentar y construir los requisitos necesarios para integrar las herramientas RULER dentro del curriculum educativo del centro. Los profesores recibieron la formación sobre las cuatro herramientas que son los pilares de la educación emocional. Los cuales, deben aplicar primero en ellos mismos e integrarlos en su vida diaria personal/profesional durante varios meses, para posteriormente enseñarlos e integralos en sus cla- 
ses. Para una descripción de herramientas, contenidos y procedimientos, ver Brackett, Rivers, Maurer, Elbertson, \& Kremenitzer, (2011). Específicamente, el entrenamiento SEL se conceptualizada en base a cinco habilidades clave conocidas bajo el acrónimo RULER (en inglés):

- Reconocer emociones para obtener información valiosa sobre el medio ambiente;

- Comprender las causas y consecuencias de las emociones de la comunidad educativa para predecir el comportamiento;

- Etiquetar las emociones para describir la experiencia emocional de manera precisa y completa;

- Expresar emociones para comunicar de manera apropiada y de acuerdo al contexto;

- Regular las propias emociones para aprovechar el poder constructivo de las emociones.

El entrenamiento de las habilidades para percibir de manera precisa emociones, tanto a nivel personal como interpersonal, comprender la información emocional y adquirir un repertorio de destrezas para manejar emociones negativas, supone una de las vías más prometedoras para tener un profesorado más positivo y efectivo en las organizaciones educativas. Sin embargo, pocas iniciativas especialmente en España, han centrado sus esfuerzos en la mejora de las competencias emocionales entre los profesores y líderes educativos, a pesar de que estas competencias han sido ampliamente relacionados con el desarrollo cognitivo, social y personal en población adulta (Mayer, Roberts y Barsade, 2008). Este método sin embargo, promueve un cambio en la cultura del centro, una integración de las herramientas pilares en el proyecto educativo del mismo, así como en los objetivos curriculares. Para una revisión reciente, descripción de la teoría del cambio, herramientas y evidencia, ver Nathanson, Rivers, Flynn y Brackett (2016).

En el presente trabajo, los profesores, todos pertenecientes al mismo centro educativo, recibieron una formación extendida a lo largo de 3 meses. La capacitación estuvo compuesta por 24 horas presenciales que se realizaron en ocho sesiones de 3 horas fuera de la jornada laboral. En esta primera fase de formación se basa en que los profesores aprendan los pilares de la educación emocinoal con cuatro herramientas (El Acuerdo emocional, el Medidor Emocional, El Meta-Momento y el Plano Emocionalmente Inteligente). Estas herramientas RULER promueven la conciencia personal y social de las emociones, mejoran la capacidad para entender y regular las propias, así como, aumentar la empatía y la toma de perspectiva. Mejo- 
ran el clima de clase y desarrollan habilidades de regulación y manejo emocional interpersonal. Para una descripción completa de las herramientas que componen el método, ver (Brackett et al., 2011).

\section{Análisis de datos}

En primer lugar, se realizaron análisis descriptivos e índices de fiabilidad de las medidas usadas. En segundo lugar, se realizaron análisis de correlaciones entre las variables de IE, compromiso laboral y burnout. Posteriormente, se realizó el análisis T-Student para cada una de las variables a nivel pre-test, con el fin de examinar si existían diferencias significativas entre los grupos experimental y control antes de la intervención.

Para evaluar la efectividad de la interveción en las habilidades de IE y las variables relacionadas con el bienestar laboral, se realizó un análisis multivariado de covarianza (MANCOVA) para cada una de las variables de interés (IE, compromiso y burnout). Este análisis estadístico permitió evaluar la efectividad inter-sujeto de la intervención en el post-test, para cada una de las variable dependientes. Por su parte, el género y las medidas pre-test fueron incluidas como covariables. Los tamaños del efecto se calcularon utilizando $\eta^{2}$ parcial. Estos datos fueron analizados con el paquete estadístico SPSS 22.0.

\section{Resultados}

\section{Análisis descriptivos e índices de fiabilidad}

Los resultados de medias y desviaciones típicas y los coeficientes de fiabilidad de las variables examinadas en este estudio se presentan en la Tabla 1.

Tabla 1. Estadísticos descriptivos e Índices de Fiabilidad

\begin{tabular}{lcccc}
\hline & N & Media & DS & $\begin{array}{c}\boldsymbol{\alpha} \text { de } \\
\text { Cronbach }\end{array}$ \\
\hline 1. Percepción emocional & 54 & 103,60 & 12,93 & 0,92 \\
2. Facilitación emocional & 54 & 99,04 & 10,07 & 0,71 \\
3. Comprensión emocional & 54 & 100,91 & 11,15 & 0,80 \\
4. Regulación emocional & 54 & 104,59 & 11,93 & 0,83 \\
5. Vigor & 54 & 5,16 & 0,66 & 0,87 \\
6. Dedicación & 54 & 5,45 & 0,57 & 0,90 \\
7. Absorción & 54 & 4,97 & 0,94 & 0,87 \\
8. Agotamiento & 54 & 2,07 & 1,00 & 0,83 \\
9. Despersonalización & 54 & 0,66 & 0,73 & 0,48
\end{tabular}


Resultados preliminares del método RULER de educación socio-emocional en las habilidades de inteligencia emocional y compromiso laboral de profesores Españoles

10. Realización Personal $\quad 54 \quad 5,05 \quad 0,56 \quad 0,90$

\section{Análisis de correlaciones}

Los resultados del análisis de correlación entre las variables de inteligencia emocional, compromiso y burnout se pueden observar en la Tabla 2.

Tabla 2. Análisis de correlación entre las diferentes variables

\begin{tabular}{|c|c|c|c|c|c|c|c|c|c|c|}
\hline & 1 & 2 & 3 & 4 & 5 & 6 & 7 & 8 & 9 & 10 \\
\hline $\begin{array}{l}\text { 1.Percepción emocio- } \\
\text { nal }\end{array}$ & $\begin{array}{ll}--- \\
\end{array}$ & $0,53 * *$ & 0,15 & $0,45 * *$ & 0,24 & $0,31 *$ & 0,03 & $-0,26$ & $-0,20$ & 0,16 \\
\hline $\begin{array}{l}\text { 2.Facilitación emo- } \\
\text { cional }\end{array}$ & & ---- & 0,21 & $0,42 * *$ & 0,26 & 0,26 & 0,08 & $-0,08$ & $-0,21$ & 0,13 \\
\hline $\begin{array}{l}\text { 3.Comprensión emo- } \\
\text { cional }\end{array}$ & & & ---- & 0,19 & 0,06 & 0,00 & 0,00 & $-0,02$ & 0,14 & $-0,05$ \\
\hline $\begin{array}{l}\text { 4. Regulación emo- } \\
\text { cional }\end{array}$ & & & & ---- & 0,23 & 0,23 & $0,27 *$ & $-0,05$ & $-0,21$ & 0,11 \\
\hline 5. Vigor & & & & & ---- & $0,61 * *$ & $0,38 * *$ & $-0,27 *$ & $-0,17$ & $0,61 * *$ \\
\hline 6. Dedicación & & & & & & ---- & $0,59 * *$ & $-0,22$ & $-0,17$ & $0,57 * *$ \\
\hline 7. Absorción & & & & & & & ---- & $-0,15$ & $-0,19$ & $0,57 * *$ \\
\hline 8. Agotamiento & & & & & & & & ---- & $0,38 * *$ & $-0,33 *$ \\
\hline 9. Despersonalización & & & & & & & & & ---- & $-0,14$ \\
\hline $\begin{array}{l}\text { 10. Realización per- } \\
\text { sonal }\end{array}$ & & & & & & & & & & ---- \\
\hline
\end{tabular}

\section{Diferencias en pre-test}

A nivel pre-test, los resultados no mostraron diferencias significativas para la variable de $I E$ [Lambda de Wilks $F(4,56)=0,06 ; p=0,99]$. Tampoco fueron encontradas diferencias significativas para la medida de compromiso laboral [Lambda de Wilks $F(3,59)=0,68 ; p=$ 0,56]. De la misma manera, los resultados tampoco mostraron diferencias entre los grupos a nivel pre-test para la variable de burnout [Lambda de Wilks $F(3,58)=2,30 ; p=0,86$ ]. Ver Tabla 3.

Tabla 3. Medidas pre-test para los grupos experimental y control 


\begin{tabular}{|c|c|c|c|c|}
\hline & \multicolumn{4}{|l|}{ Pre-test } \\
\hline & \multirow{2}{*}{\multicolumn{2}{|c|}{$\begin{array}{l}\text { RULER } \\
(n=32)\end{array}$}} & \multirow{2}{*}{\multicolumn{2}{|c|}{$\begin{array}{l}\text { control } \\
(n=22)\end{array}$}} \\
\hline & & & & \\
\hline & $M$ & $D S$ & $M$ & $D S$ \\
\hline \multicolumn{5}{|l|}{ Inteligencia Emocional } \\
\hline Percepción & 103,73 & 2,06 & 102,58 & 2,55 \\
\hline Facilitación & 99,64 & 1,66 & 98,79 & 2,06 \\
\hline Comprensión & 101,95 & 1,95 & 102,33 & 2,42 \\
\hline Regulación & 105,11 & 1,98 & 105,37 & 2,46 \\
\hline \multicolumn{5}{|l|}{ Compromiso } \\
\hline Vigor & 4,96 & 0,14 & 5,24 & 1,97 \\
\hline Dedicación & 5,32 & 0,14 & 5,42 & 0,19 \\
\hline Absorción & 4,85 & 0,17 & 5 & 0,24 \\
\hline \multicolumn{5}{|l|}{ Burnout } \\
\hline Agotamiento Emocional & 1,8 & 0,16 & 2,38 & 0,2 \\
\hline Despersonalización & 0,55 & 0,12 & 0,81 & 0,15 \\
\hline Realización Personal & 4,82 & 0,14 & 5,08 & 0,17 \\
\hline
\end{tabular}

\section{Efectividad del método}

La Tabla 4 muestra las medias y desviación típica en el post-test. Con el objetivo de testar la efectividad en el tiempo 2 (post-test) entre el grupo experimental y control, se llevó a cabo un análisis MANCOVA. Los resultados demuestran que los profesores que recibieron el entrenamiento RULER -en comparación con los profesores del grupo control- obtuvieron puntuaciones significativamente mayores en las habilidades de $I E$ [Lambda de Wilks $F(4,43)$ $\left.=7,95 ; p=0,00 ; \eta^{2}=0,42\right]$, en compromiso [Lambda de Wilks $F(3,46)=6,85 ; p=0,00 ; \eta^{2}$ $=0,31]$ y en burnout [Lambda de Wilks $\left.F(3,47)=3,60 ; p=0,02 ; \eta^{2}=0,19\right]$. En concreto, los contrastes univariados indican que los profesores que recibieron entrenamiento, obtuvieron puntuaciones más altas en las dimensiones de percepción, comprensión y regulación emocional, aunque el efecto no fue significativo para la dimensión de facilitación emocional, en comparación al grupo control. Por otro lado, el entrenamiento fue efectivo para todas las dimensiones de compromiso, vigor, dedicación y absorción, así como para la dimensión positiva de burnout, la realización personal en el trabajo. Sin embargo, no se obtuvo efecto significativo en la variable de despersonalización, ni en la reducción de los niveles de agotamiento emocional.

Tabla 4. Diferencias post-intervención grupo experimental vs control 


\begin{tabular}{|c|c|c|c|c|c|c|c|}
\hline & & \multicolumn{2}{|l|}{$\begin{array}{l}\text { RULER } \\
(n=32)\end{array}$} & \multicolumn{2}{|c|}{$\begin{array}{l}\text { control } \\
(n=22)\end{array}$} & \multirow[b]{2}{*}{$p$} & \multirow[b]{2}{*}{$\eta^{2}$} \\
\hline & & $M$ & $D T$ & $M$ & $D T$ & & \\
\hline \multicolumn{8}{|c|}{ Inteligencia Emocional } \\
\hline & Percepción & 101,48 & 13,24 & 92,66 & 14,74 & 0,008 & 0,14 \\
\hline & Facilitación & 93,1 & 9,75 & 90,45 & 14,45 & 0,343 & 0,02 \\
\hline & Comprensión & 106,65 & 10,35 & 89,62 & 14,5 & 0,001 & 0,37 \\
\hline & Regulación & 107,2 & 12,66 & 95,33 & 17,33 & 0,004 & 0,17 \\
\hline \multicolumn{8}{|l|}{ Compromiso } \\
\hline & Vigor & 5,21 & 1,09 & 4,59 & 0,91 & 0,001 & 0,22 \\
\hline & Dedicación & 5,34 & 1,21 & 4,6 & 0,88 & 0,001 & 0,23 \\
\hline & Absorción & 4,94 & 1,17 & 4,49 & 0,97 & 0,044 & 0,07 \\
\hline \multicolumn{8}{|l|}{ Burnout } \\
\hline & $\begin{array}{l}\text { Agotamiento Emo- } \\
\text { cional }\end{array}$ & 1,73 & 0,89 & 2,43 & 0,93 & 0,114 & 0,05 \\
\hline & Despersonalización & 0,52 & 0,66 & 1,07 & 0,93 & 0,054 & 0,08 \\
\hline & Realización Personal & 5 & 1,06 & 4,56 & 1,25 & 0,018 & 0,11 \\
\hline
\end{tabular}

Nota: Género, edad, y las medidas pre-test fueron usadas como covariables en todos los análisis

\section{Discusión y Conclusiones}

El presente trabajo proporciona apoyo empírico acerca del impacto positivo que un programa SEL, el método RULER, tiene en las habilidades de percepción, comprensión y regulación emocional, así como en la promoción de aspectos relacionados con la satisfacción y el rendimiento laboral en una muestra de profesores españoles, en comparación con un grupo control equivalente que no recibió el entrenamiento. Nuestros resultados apoyan investigaciones previas que muestran como las habilidades de IE confieren recursos y estrategias para hacer frente a las altas demandas emocionales, permitiendo mayor satisfacción, bienestar y una mejor calidad de las interacciones, particularmente en profesorado (Brackett, Palomera, Mojsa-Kaja , Reyes y Salovey, 2010; Saklofske et al., 2012). Los resultados obtenidos apoyan parciamente nuestras hipótesis, por un lado podemos concluir que el método RULER impacta positivamente en las competencias de IE, concretamente para comprender la información emocional y el entendimiento de las causas y consecuencias de las emociones, el uso de un vocabulario emocional amplio, así como la adquisición de un repertorio de destrezas para manejar las emociones. Por otro lado, parece que frena la disminución de la percepción emocional, y ha sido efectivo para la promoción de variables laborales positivas (por ej., los sentimientos de realización personal, de logro y eficacia mientras se trabaja, así como todas las variables relacionadas con el compromiso laboral, tales como la energía, la concentración, el 
orgullo y la motivación en el desempeño profesional). Por lo que facilitó un mayor bienestar emocional y compromiso laboral, que el grupo de comparación. No obstante, no se obtuvieron diferencias significativas en los niveles de burnout. Parece que este hecho pueda ser debido a que los profesores empezaran a incluir en sus prácticas educativas, herramientas que permitían el fomento de la consciencia personal y social, la promoción de un clima educativo positivo y el manejo efectivo de los conflictos. El uso de este tipo de herramientas durante el curso académico hace pensar que facilitara mayores oportunidades de trabajo grupal e interacciones sociales más positivas entre alumnos y profesorado, así como una mayor efectividad en la gestión, percepción y manejo de las emociones por parte de los docentes, lo cual es determinante para favorecer una percepción subjetiva mayor de su realización, eficacia, mayor tolerancia al estrés, afecto positivo mientras se trabaja, mejores competencias socioemocionales y ajuste emocional (Brackett et al., 2010; Kurki, et al., 2016; Rey et al., 2016; Yin et al., 2013).

No obstante, es preciso señalar que, en vista de los resultados obtenidos, las diferencias obtenidas señalan que el método RULER consiguió aumentar las habilidades de IE y las variables relacionas con el bienestar laboral en el grupo que recibió el entrenamiento SEL, mientras que en el grupo control, la puntuación en dichas variables decreció. Concretamente, la percepción emocional disminuyó ligeramente en el grupo RULER, pero cayó de manera bastante acusada en el grupo control; en la dimensión de facilitación emocional ambas medidas decrecieron, aunque la disminución fue más acusada de nuevo en el grupo control; en cuanto a la comprensión emocional, hubo un aumento de casi cinco puntos, mientras hubo un decrecimiento en el grupo control, por último, la regulación emocional aumentó en dos puntos en el grupo experimental, mientras disminuyó en el grupo control. De la misma manera, estos resultados también se vieron reflejados en las variables laborales en las que se obtuvieron diferencias significativas entre ambas condiciones. Concretamente, todas las variables relacionadas con el compromiso laboral y la satisfacción aumentaron ligeramente en el grupo experimental, mientras que el grupo control reportó menor percepción de bienestar laboral. Es por tanto, esta evolución diferencial en cada uno de los dos grupos a lo largo de un año académico, la que hace que las diferencias sean particularmente significativas. En base a estos resultados, podemos atribuir que el método RULER fundamentalmente consiguió desarrollar las variables objeto de estudio durante un curso académico, pero en algunos casos frenando su decrecimiento. No obstante, este hecho puede ser debido a que dado que la evaluación posttest se realizó un año académico más tarde, existe una tendencia general al decrecimiento de 
las variables objeto de estudio, a medida que aumenta el ciclo formativo de los estudiantes (Anaya y López, 2014; Anaya y López 2015). Por lo que podemos asumir, que RULER impediría y reduciría en algunos casos, ese decrecimiento.

En vista de los resultados, el método RULER muestra evidencia acerca de su efectividad en población adulta. Sin embargo, parece que existen algunas competencias que son más susceptibles de ser entrenadas o mejoradas que otras. En este trabajo, hay dos ramas de IE que no obtuvieron un incremento, la percepción y facilitación emocional. Esto puede ser debido a la naturaleza de las propias dimensiones. En primer lugar, hay ciertas críticas a la rama de percepción emocional, evaluada con el MSCEIT, que podrían justificar los resultados obtenidos, esto es, la ausencia de tareas en el cuestionario, que evalúan la percepción emocional a nivel individual, o el uso de tareas que miden la habilidad mediante fotografías estáticas o diseños artísticos, lo cual parece no ser susceptible a cambios sustanciales en esta rama, tal y como los propios autores han reconocido (Mayer et al., 2008). Por otro lado, es posible que para el desarrollo de la rama de facilitación se requiera un uso más continuado de las herramientas del método, con el fin de poder aumentar la capacidad para usar las emociones en determinados procesos cognitivos, la toma de decisiones o las relaciones. Es de suponer, pues, que los efectos en estas ramas puedan hacerse visibles en sucesivas evaluaciones de seguimiento. Es más, esta ausencia de resultados concuerda con recientes investigaciones en población adulta (Pool y Qualter, 2012). Parece, por tanto, que cuando se valoran los efectos de una intervención, los resultados en la rama de facilitación emocional no son tan fácilmente identificables (MacCann, Fogarty, Zeidner, y Roberts, 2011).

Por otro lado, la ausencia de resultados encontrados con respecto a la reducción de los niveles de burnout, puede ser debido a que la muestra de profesorado que ha participado en este estudio no contara con niveles suficientes de desgaste o estrés laboral, por lo que se hace muy difícil poder obtener cierta efectividad del entrenamiento si se parte de niveles muy bajos en esta dimensión (Pena y Extremera, 2012; Pena, Rey y Extremera, 2012). Concretamente, la dimensión de despersonalización es una variable especialmente controvertida cuando se evalúa entre profesionales docentes. De hecho, en nuestra muestra de profesorado, cuenta con un valor reducido de alpha $=0,48$. Por tanto, la ausencia de efecto puede estar en relación al propio sesgo de deseabilidad social en referencia a ítems que califican a los alumnos como si fueran objetos, la actitud cínica o la insensibilidad. 


\section{Limitaciones y líneas futuras}

La limitación más importante del presente trabajo es el tamaño de la muestra y la no aleatorización a nivel de los participantes. La naturaleza propia del estudio, las dificultades de acceso a la muestra, así como de centros que permitan la aplicación del método con una implicación importante de su equipo directivo y su profesorado, son algunas de las razones. La inclusión de un mayor número de participantes que equilibrara la cantidad de hombres y mujeres en el entrenamiento, hubiera permitido explorar la existencia de diferencias en función del género. Adicionalmente, la participación de un solo colegio, tanto experimental como control, no permite generalizar los resultados a otras muestras españolas, por lo que la participación de otros colegios hubiera permitido comparar la efectividad de la intervención en diferentes profesionales provenientes de otros contextos educativos (públicos, concertados) y de otras zonas socio-económicas. Otra limitación importante, es que el centro educativo que sirvió como grupo control para comparar la efectividad del entrenamiento, no recibieron ningún tipo de formación en ese momento, por lo que la comparación con otra formación equivalente, hubiera sido útil para comparar y testar la efectividad del método. Una tercera limitación es el uso de medidas auto-informadas, en lo que a las variables laborales se refiere. Por ello, como prospectiva de investigación se sugiere la inclusión de medidas más objetivas de rendimiento profesional, satisfacción y compromiso laboral, así como la evaluación por parte de compañeros, estudiantes y/o entorno profesional. Asímismo, se sugiere evaluar la efectividad de este tipo de iniciativas a medio y largo plazo, incluyendo medidas de seguimiento a partir de los 3/6 meses, con el fin de evaluar la estabilidad de los resultados. Adicionalmente, sería interesante medir el impacto los estudiantes con la inclusión de variables de rendimiento académico, ajuste psico-social o clima escolar, con el fin de testar las implicaciones distales de la educación SEL en la comunidad educativa en general. De hecho, hay una amplia evidencia científica que muestra el impacto que variables relacionadas con la satisfacción o el compromiso laboral docente puede tener en el clima que los estudiantes necesitan experimentar para su óptimo rendimiento e incluso desarrollo. La ausencia de estas variables, puede tener implicaciones importantes en el rendimiento académico, motivación e incluso la salud mental del alumnado (Oberle y Schonert-Reichl, 2016; Reyes, Brackett, Rivers, White y Salovey, 2012).

Este estudio pretende contribuir al avance en el conocimiento acerca del impacto del desarrollo efectivo de las habilidades de IE en población adulta. Por el momento, en nuestro país, son pocas las iniciativas que han centrado sus esfuerzos en valorar la efectividad de los 
programas SEL. A pesar de su ampliamente mostrada relevancia en el contexto educativo, sigue siendo escaso el estudio de la efectividad de estas iniciativas entre los profesores y líderes educativos. Además, nuestra investigación prueba la efectividad de un programa de entrenamiento científicamente sustentado en el modelo de habilidad de la IE (Brackett y Rivers, 2013). La aplicación de un entrenamiento científicamente estructurado y la utilización de medidas de ejecución y medidas auto-informadas -ampliamente validadas en contexto español-, representan dos puntos fuertes en el presente trabajo. Los resultados ponen de manifiesto que aquellos profesores que recibieron formación en IE incrementaron significativamente sus habilidades de IE (comprensión y regulación emocional) medidas mediante tareas de ejecución (MSCEIT), así como su satisfacción y compromiso laboral. Los resultados obtenidos suponen una de las vías más prometedoras para favorecer una mejora en el funcionamiento laboral, personal y social en la población docente, dada su influencia en el desarrollo de entornos de enseñanza/aprendizaje más productivos y un ambiente de clase más óptimo (Aloe et al., 2014). En conclusión, el entrenamiento SEL puede ser una herramienta clave para la promoción del bienestar laboral y el desarrollo profesional en el profesorado de contextos educativos españoles.

\section{Referencias}

Anaya, D. y López, E. (2015). Satisfacción laboral del profesorado de educación secundaria. Revista de Investigación Educativa, 33(2), 435-452. doi:10.6018/rie.33.2.202841

Anaya, D. y López, E. (2014): Satisfacción laboral del profesorado en 2012-13 y comparación con los resultados de 2003-04. Un estudio de ámbito nacional. Revista de Educación, 365, 96-121. doi:10.4438/1988-592X-RE-2014-365-266

Aloe, A. M., Amo, L. C., \& Shanahan, M. E. (2014). Classroom management self-efficacy and burnout: A multivariate meta-analysis. Educational Psychology Review, 26(1), 101-126. doi:10.1007/s10648-013-9244-0

Bakker, A. B., Hakanen, J. J., Demerouti, E., \& Xanthopoulou, D. (2007). Job resources boost work engagement, particularly when job demands are high. Journal of Educational Psychology, 99(2), 274. doi: 10.1037/0022-0663.99.2.274

Bar-On, R. (2006). The Bar-On model of emotional-social intelligence (ESI) 1. Psicothema, 18(Suplemento),

Disponible

en:<http://tuxchi.redalyc.org/articulo.oa?id=72709503> ISSN 0214-9915 
Betoret, F. D. (2009). Self-efficacy, school resources, job stressors and burnout among Spanish primary and secondary school teachers: a structural equation approach. Educational Psychology, 29, 45-68. doi:10.1080/01443410802459234.

Blanco-Donoso, L. M., Demerouti, E., Hernández, E. G., Moreno-Jiménez, B., \& Cobo, I. C. (2015). Positive benefits of caring on nurses' motivation and well-being: A diary study about the role of emotional regulation abilities at work. International Journal of Nursing studies, 52(4), 804-816. doi: 10.1016/j.ijnurstu.2015.01.002

Brackett, M. A., Rivers, S. E., Maurer, M., Elbertson, N. A., \& Kremenitzer, J. P. (2011). Creating emotionally literate learning environments. In M. A. Brackett, J. P. Kremenitzer, M. Maurer, S. E. Rivers, N. A. Elberston \& M. D. Carpenter (Eds.), Creating emotionally literate learning environments (pp. 1-21). Port Chester, NY: National Professional Resources, Inc.

Brackett, M. A., Palomera, R., Mojsa-Kaja, J., Reyes, M. R., \& Salovey, P. (2010). Emotion-regulation ability, burnout, and job satisfaction among British secondary-school teachers. Psychology in the Schools, 47(4), 406-417. doi:10.1002/pits.20478

Brackett, M. A., \& Rivers, S. E. (2013). Transforming students' lives with social and emotional learning. In R. Pekrun \& L. Linnenbrink-Garcia (Eds.), International handbook of Emotions in Education (pp. 368-388). New York, NY: Taylor and Francis.

Brackett, M. A., Rivers, S. E., Reyes, M. R., \& Salovey, P. (2012). Enhancing academic performance and social and emotional competence with the RULER feeling words curriculum. Learning and Individual Differences, 22(2), 218-224. doi: 10.1016/j.lindif.2010.10.002

Castillo, R., Fernández-Berrocal, P., \& Brackett, M. A. (2013). Enhancing teacher effectiveness in Spain: A pilot study of the RULER approach to social and emotional learning. Journal of Education and Training Studies, 1(2), 263-272. doi:10.11114/jets.v1i2.203

Castillo, R., Salguero, J. M., Fernández-Berrocal, P., \& Balluerka, N. (2013). Effects of an emotional intelligence intervention on aggression and empathy among adolescents. Journal of Adolescence, 36(5), 883-892. doi: 10.1016/j.adolescence.2013.07.001

Chang, M. L. (2013). Toward a theoretical model to understand teacher emotions and teacher burnout in the context of student misbehavior: Appraisal, regulation and coping. Motivation and Emotion, 37(4), 799-817. doi: 10.1007/s11031-012-9335-0

De Jonge, J., \& Dormann, C. (2006). Stressors, resources, and strain at work: a longitudinal test of the triple-match principle. Journal of Applied Psychology, 91(6), 1359. doi: 10.1037/0021-9010.91.5.1359 
Downey, L. A., Johnston, P. J., Hansen, K., Birney, J., \& Stough, C. (2010). Investigating the mediating effects of emotional intelligence and coping on problem behaviours in adolescents. Australian Journal of Psychology, 62(1), 20-29. doi: 10.1080/00049530903312873

Durlak, J. A., Domitrovich, C. E., Weissberg, R. P., \& Gullotta, T. P. (Eds.). (2015). The handbook of social and emotional learning. New York, NY: The Guilford Press.

Durlak, J. A., Weissberg, R. P., Dymnicki, A. B., Taylor, R. D., \& Schellinger, K. B. (2011). The impact of enhancing students' social and emotional learning: A meta-analysis of school-based universal interventions. Child development, 82(1), 405-432. doi:10.1111/j.1467-8624.2010.01564.x

Extremera, N., \& Fernández-Berrocal, P. (2009). Test de Inteligencia Emocional de Mayer Salovey Caruso. Madrid: TEA Ediciones.

Extremera, N., \& Rey, L. (2016). Ability emotional intelligence and life satisfaction: Positive and negative affect as mediators. Personality and Individual Differences, 102, 98-101. doi: 10.1016/j.paid.2016.06.051

Gardner, H. (1983): Frames of Mind. The Theory of Multiple Intelligences. New York, Basic Books.

Hagelskamp, C., Brackett, M. A., Rivers, S. E., \& Salovey, P. (2013). Improving classroom quality with the ruler approach to social and emotional learning: Proximal and distal outcomes. American Journal of Community Psychology. doi: 10.1007/s10464-0139570-x

Jennings, P. A., \& Greenberg, M. T. (2009). The prosocial classroom: Teacher social and emotional competence in relation to student and classroom outcomes. Review of Educational Research, 79(1), 491-525. doi:10.3102/0034654308325693

Kagan, S. (1992). Cooperative learning. San Juan Capistrano, CA: Resources for Teachers.

Kurki, K., Järvenoja, H., Järvelä, S., \& Mykkänen, A. (2016). How teachers co-regulate children's emotions and behaviour in socio-emotionally challenging situations in day-care settings. International Journal of Educational Research, 76, 76-88. doi:10.1016/j.ijer.2016.02.002

MacCann, C., Fogarty, G. J., Zeidner, M., \& Roberts, R. D. (2011). Coping mediates the relationship between emotional intelligence (EI) and academic achievement. Contemporary Educational Psychology, 36(1), 60-70. doi: 10.1016/j.cedpsych.2010.11.002 
Mayer, J. D., Roberts, R. D., y Barsade, S. G. (2008). Human Abilities: Emotional Intelligence. Annual Review of Psychology, 59, 507-536. doi:10.1146/annurev.psych.59.103006.093646

Mayer, J. D., y Salovey, P. (1997). What is emotional intelligence? En P. Salovey y D. J. Sluyter (coords.), Emotional development and emotional intelligence: Educational implications. (pp. 3-34). New York, NY US: Basic Books.

Mayer, J. D., Salovey, P., \& Caruso, D. (2002). Mayer-Salovey-Caruso Emotional Intelligence Test (MSCEIT® V2.0): User's manual. Toronto, ON: Multi-Health Systems.

Maslach, C., Jackson, S., y Leiter, M. P. (1996). Maslach Burnout Inventory (3rd ed.). Palo Alto,CA: Consulting Psychologists Press (Versión en castellano). Madrid: TEA Ediciones).

Nathanson, L., Rivers, S. E., Flynn, L. M., \& Brackett, M. A. (2016). Creating emotionally intelligent schools with RULER. Emotion Review, 8(4), 1-6. doi:10.1177/1754073916650495

Oberle, E., \& Schonert-Reichl, K. A. (2016). Stress contagion in the classroom? The link between classroom teacher burnout and morning cortisol in elementary school students. Social Science \& Medicine, 159, 30-37. doi: 10.1016/j.socscimed.2016.04.031

Pena, M. y Extremera, N. (2012). Inteligencia emocional percibida en profesorado de Primaria y su relación con los niveles de burnout e ilusión por el trabajo (engagement). Revista de Educación. 359, 604-627. doi: 10-4438/1988-592X-RE-2010-359-109

Pena, M., Rey, L. y Extremera, N. (2012). Bienestar personal y laboral en el profesorado de Infantil y Primaria: Diferencias en función de su inteligencia emocional y del género. Revista de Psicodidáctica, 17 (2), 341-360. doi: 10.1387/Rev.Psicodidact.4496

Pérez-Escoda, N., Filella, G., Bisquerra, R., \& Alegre, A. (2012). Desarrollo de la competencia emocional de maestros y alumnos en contextos escolares [Development of emotional competence of teachers and students in school contexts]. Electronic Journal of Research in Educational Psychology, 10(3), 1183-1208. Disponible en:<http://www.redalyc.org/articulo.oa?id=293124654012>

Petrides, K. V., \& Furnham, A. (2003). Trait emotional intelligence: Behavioural validation in two studies of emotion recognition and reactivity to mood induction. European Journal of Personality, 17(1), 39-57. doi: 10.1002/per.466 
Pool, L. D., \& Qualter, P. (2012). Improving emotional intelligence and emotional selfefficacy through a teaching intervention for university students. Learning and Individual Differences, 22(3), 306-312. doi:10.1016/j.lindif.2012.01.010

Rey, L., Extremera, N., \& Pena, M. (2016). Emotional competence relating to perceived stress and burnout in Spanish teachers: a mediator model. Peer Journal, 4, e2087. doi: 10.7717/peerj.2087

Reyes, M. R., Brackett, M. A., Rivers, S. E., White, M., \& Salovey, P. (2012). Classroom emotional climate, student engagement, and academic achievement. Journal of Educational Psychology, 104(3), 700-712. doi:10.1037/a0027268

Reyes, M. R., Brackett, M. A., Rivers, S. E., Elbertson, N. A., \& Salovey, P. (2012). The interaction effects of program training, dosage, and implementation quality on targeted student outcomes for the RULER approach to social and emotional learning. School Psychology Review, 41(1), 82.

Roeser, R. W., Skinner, E., Beers, J., \& Jennings, P. A. (2012). Mindfulness training and teachers' professional development: An emerging area of research and practice. Child Development Perspectives, 6(2), 167-173. doi:10.1111/j.1750-8606.2012.00238.x

Ruiz-Aranda, D., Castillo, R., Salguero, J. M., Cabello, R., Fernández-Berrocal, P., \& Balluerka, N. (2012). Short-and midterm effects of emotional intelligence training on adolescent mental health. Journal of Adolescent Health, 51(5), 462-467. doi: 10.1016/j.jadohealth.2012.02.003

Saklofske, D. H., Austin, E. J., Mastoras, S. M., Beaton, L., \& Osborne, S. E. (2012). Relationships of personality, affect, emotional intelligence and coping with student stress and academic success: Different patterns of association for stress and success. Learning and Individual Differences, 22(2), 251-257. doi:10.1016/j.lindif.2011.02.010

Sanchez-Garcia, M., Extremera, N., \& Fernandez-Berrocal, P. (2016, January 11). The Factor Structure and Psychometric Properties of the Spanish Version of the Mayer-SaloveyCaruso Emotional Intelligence Test. Psychological Assessment. Advance online publication. doi: 10.1037/pas0000269

Schaufeli, W. B., Salanova, M., González-Romá, V., y Bakker, A. B. (2002). The measurement of engagement and burnout: A two sample confirmatory factor analytic approach. Journal of Happiness Studies, 3(1), 71-92. doi: 10.1023/A:1015630930326

Talvio, M., Lonka, K., Komulainen, E., Kuusela, M., \& Lintunen, T. (2013). Revisiting Gordon's Teacher Effectiveness Training: An intervention study on teachers' social and 
emotional learning. Electronic Journal of Research in Educational Psychology, 11(3), 693-716. doi:10.14204/ejrep.31.13073

Yin, H. B., Lee, J. C. K., \& Zhang, Z. H. (2013). Exploring the relationship among teachers' emotional intelligence, emotional labor strategies and teaching satisfaction. Teaching and Teacher Education, 35, 137-145. doi: 10.1016/j.tate.2013.06.006 